LOWE, C. F. \& CHADWICK, P. J. (1990) Verbal control of delusions. Behaviour Therapy, 21, 461-480.

David Healy Academic Subdepartment of Psychological Medicine Denbigh, Clwyd LL16 5SS

\section{Clozapine and negative symptoms}

SIR: I read with interest about the remarks made by Healy (Journal, January 1993, 162, 23-29) on the superiority of clozapine over traditional neuroleptics and would like to express some reservations about this drug's ability to improve the negative symptoms of schizophrenia. Negative symptoms are ill-defined, and lack firmly established construct validity; they may be mimicked by drug-induced akinesia and depression (de Leon, 1989). The apparent improvement of negative symptoms in Kane et als (1988) study may be for a number of reasons. These include the reduction of positive symptoms, the use of a nonspecific measurement scale (Brief Psychiatric Rating Scale), the relief of depression in chronic psychotic patients (clozapine is suspected of having some antidepressant property), and diminution of extrapyramidal side-effects. The latter possibility was supported by the significantly lower scores for extrapyramidal side-effect of the clozapine group than the chlorpromazine group from week four until the end of the trial. Perhaps a better way to prove clozapine's superiority in improving negative symptoms would be a controlled trial of treatment for 'pure negative syndrome', simple schizophrenia, or the residual negative syndrome. Until then we should be sceptical of its specificity in improving negative symptoms.

DE LeON, J., Wilson, W. H. \& Simpson, G. M. (1989) Measurement of negative symptoms in schizophrenia. Psychiatric Development. 3, 211-234.

KANe, J., Honigfeld, G., Singer, J., et al (1988) Clozapine for the treatment-resistant schizophrenic. Archives of General Psychiatry, 45, 789-796.

Prince of Wales Hospital

YUN-KwoK WING

Chinese University of Hong Kong

Shatin, NT

Hong Kong

\section{Neuroleptic rechallenge after neuroleptic malignant syndrome}

SIR: We read with interest the report by Weller \& Kornhuber (Journal, December 1992, 161, 855-856) on the use of the atypical antipsychotic clozapine after an episode of neuroleptic malignant syndrome (NMS). Being also concerned with the clinical man- agement and follow-up of this syndrome, we feel it necessary to raise some points regarding the question of whether and which neuroleptic drug could be readministered as a further treatment of psychotic symptoms.

We would like to emphasise that the expression 'clozapine rechallenge' which is notified in the title of the article could lead to a potentially harmful misinterpretation. Indeed, the term rechallenge is defined as the giving of a further dose of a drug to a patient who had previously taken the same drug and in whom an adverse event, which might be due to that drug, had subsequently occurred (Stephens, 1988). Whether accidental or deliberate, the rechallenge is generally considered a major criterion in the assessment of an adverse drug reaction. The authors administered clozapine for persistent psychiatric symptoms to patients who had previously developed NMS when treated with prior neuroleptics, mainly phenothiazines and butyrophenones, but not clozapine. It is thus misleading to suggest that clozapine has been readministered after recovery of the NMS episode when this drug has just been chosen as a further and alternative antipsychotic medication. On the other hand, the authors suggest that clozapine should be considered a drug of choice for psychotic patients with a history of NMS.

However, it has been reported that clozapine by itself may be implicated in the development of NMS (Miller et al, 1991; DasGupta \& Young, 1991). Furthermore, additional cofactors are required for the development of NMS and a number of patients may successfully tolerate careful neuroleptic reexposure after at least two weeks of complete recovery of the index episode (Pope et al, 1991). How to treat subsequent psychotic manifestations in a patient after an episode of NMS is a matter of increasing attention among clinicians, and nonneuroleptic treatments such as ECT have also been considered (Bottaï et al, 1992). Despite the distinctive pharmacological profile of clozapine among antipsychotic drugs, it may be premature to speculate about the peculiar interest of this drug in the further management of patients with a history of NMS.

Bottaï, T., Petit, P., Pinzani, V., et al (1992) Neuroleptic malignant syndrome: 11 case reports. 18 th Collegium Internationale Neuropsychopharmacologicum. Clinical Neuropharmacology. 15 (suppl. 1), 138B.

DasGuPTA, K. \& Young, A. (1991) Clozapine-induced neuroleptic malignant syndrome. Journal of Clinical Psychiatry, 52, 105-107.

Miller, D. D., Sharafuddin, M. J. \& Kathol, R. G. (1991) A case of clozapine-induced neuroleptic malignant syndrome. Journal of Clinical Psychiatry, 52, 99-101.

PoPe, H. G., AIZley, H. G., KeCk, P. E., et al (1991) Neuroleptic malignant syndrome: long-term follow-up of 20 cases. Journal of Clinical Psychiatry, 52, 208-212. 